\title{
A Robust Method for Moving Object Detection Using Modified Statistical Mean Method
}

\author{
Safvan Vahora ${ }^{1}$, Narendra Chauhan ${ }^{2}$, and Nilesh Prajapati ${ }^{3}$ \\ ${ }^{1}$ Dept. of Information Technology, Vishvakarma Government Engineering College, \\ Ahmedabad, India \\ ${ }^{2}$ Dept. of Information Technology, A. D. Patel Institute of Technology, Anand, India \\ ${ }^{3}$ Dept. of Information Technology, Birla Vishvakarma Mahavidyalaya, Anand, India \\ \{safvan465, narendracchauhan\} @gmail.com, \\ nilesh.prajapati@bvmengineering.ac.in
}

\begin{abstract}
.
Moving object detection is low-level, important task for any visual surveillance system. One of the aim of this paper is to, to describe various approaches of moving object detection such as background subtraction, temporal difference, as well as pros and cons of these techniques. A statistical mean technique [10] has been used to overcome the problem in previous techniques. Even statistical mean method also suffers with the problem of superfluous effects of foreground objects. In this paper, the presented method tries to overcome this effect as well as reduces the computational complexity up to some extent. In this paper, a robust algorithm for automatic, noise detection and removal from moving objects in video sequences is presented. The algorithm considers static camera parameters.
\end{abstract}

\section{Keywords:}

Moving object detection, Noise detection and removal, Statistical mean technique.

\section{Introduction}

Computer vision system have been developed, in order to simulate the most natural systems which have ability to deal with changing environments such as moving objects, objects tracking, changing illumination, and changing view point. In video surveillance system, detection and tracking of object is lower level task that provides support to higher level tasks such as event detection. Categorizing moving objects is a critical task which requires video segmentation, which is used in number of computer vision applications such as video surveillance, traffic monitoring, and remote sensing [1]. There are three major steps in video surveillance analysis: detection of moving objects, tracking of interested objects from consecutive frames, and the third is analysis of these tracked objects to identify its behavior, and also to identify normal/abnormal events.

There are many applications those uses video surveillance. Video traffic monitoring is gathering traffic information from various visual sources to redirect the traffic flow. Smart video surveillance system is monitoring scene continuously, to detect a desired event. Gesture recognition is to identify human gesture, fingerprint detection and eyes detection to login into a system. Video indexing can be used for automatically explanation and retrieval of videos in multimedia database. 
Moving object detection process of classifying the pixels in video frames into the two classes, foreground pixels are considered as pixels of moving object and background pixels are considered as pixels of stationary background object. In another words, moving object detection handles segmentation of from stationary background object.

Detection of moving object can be complex due to several reasons such as noise in video sequence, sudden illumination changes, shadows and waving tree branches in the wind, rain or snow-fall for outdoor video surveillance, occlusion provide loss of information by projection of $3 \mathrm{D}$ world in 2D image [9].

Different approaches have been proposed by different researchers for moving object detection. In this paper, a modified statistical mean technique has been presented for moving object detection, that having various advantages over the traditional approaches of moving object detection. The presented method tries to reduce the effect of noise found during the detection of moving objects. Hence it increases the robustness in detection process.

Figure 1 shows the framework of presented robust approach for moving object detection by noise detection and removal. We describe each phase in subsequent section.

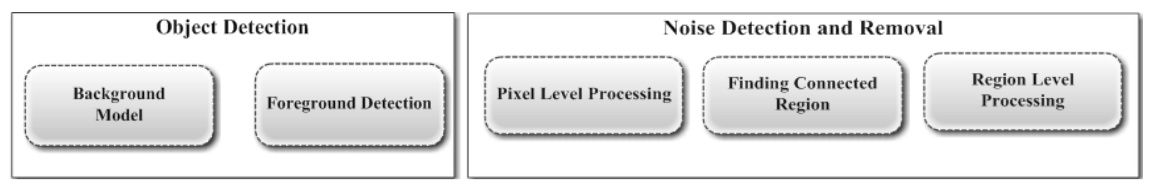

Figure 1: Framework of Noise Robust detection of moving object

The rest of the paper is organized as follows. In section 2, we give details of traditional background subtraction technique, temporal difference and statistical mean technique of moving object detection. In section 3, we describe a robust method for noise detection and removal during the detection of moving objects. This has been achieved by using modification to statistical mean technique which is the modified version of statistical mean technique as described in section 2 . Experimental results and conclusion are described in section 4 and section 5 respectively.

\section{Methods for Moving Object Detection}

\subsection{Background Subtraction}

Object detection $[3,6,7,8,10]$ can be achieved by creating background model and then finding deviations from the model for each and every frame in video. Background subtraction is widely used because of its time complexity less for detecting the foreground object. Background subtraction is particularly a for motion segmentation in static scenes [2].

Background subtraction is most popular choice to detect stationary foreground objects [6,7], because they work well when the camera is static and illumination change is gradual [8].

In this method, to detect the foreground object, the background subtraction algorithm detects the difference between the current image and the background image or often called reference image or background model. Recent background subtraction algorithms focused on robust background modeling and updating to adapt to varying illumination condition between night and day, light effects, background structures, background change from whether change and repetitive motion from clutter [3]. For a pixel location (x, y) in the current image I, is identified as foreground pixel along with background image $\mathrm{B}$, if it satisfies the equation 1 . 
International Journal of Advanced Information Technology (IJAIT) Vol. 2, No.1, February 2012

$$
|I(x, y)-B(x, y)|>\tau
$$

where $\tau$ is a predefined threshold [4]. The background image B is updated by the adaption coefficient $\eta$ as per the equation 2 .

$$
B_{i+1}=\eta I_{i}+(1-\eta) B_{i}
$$

The core part of this method is to integrate the new upcoming information of a new image into the background image, so that background image becomes more robust. The value of $\eta$ should not be large, it may create faster new changes in the background image.

The pixels constituting the regions undergoing change are marked for further processing. In general, a connected component algorithm is used to obtain connected regions corresponding to the objects.

Even though the background subtraction technique is well to extract moving object, this background subtraction technique may suffer to dynamic background changes such as the entrance of new background object or sudden illumination change.

\subsection{Temporal Differencing}

Temporal differencing technique uses the pixel wise difference between two or three frames in frame sequences to extract a moving object. [5, 10] Temporal differencing technique is computationally simple and fast as well as is adaptive to dynamic environment. In temporal difference technique, extraction of moving pixel is simple and fast. It takes difference of the current and previous frames as shown in equation 3

$$
\left|I_{i}(x, y)-I_{i-1}(x, y)\right|>\tau
$$

where $\tau$ is predefined threshold.

Temporal differencing technique is less effective in extracting all information about target object, especially when the target object has uniform texture or target object moves slowly. When a foreground object moves slowly or stops moving in between, temporal difference technique fails in detection a change between consecutive frames and loses the target object information. Temporal difference is most sensitive

to the threshold value $\tau$, to determining pixel-wise difference between consecutive frames as well as it may left holes in foreground objects. Temporal difference require special supportive algorithm to detect stopped objects.

\subsection{Statistical Mean Technique}

There are some drawbacks of traditional moving object detection approach, as described in the above section. To overcome these shortcomings, we propose the next technique is statistical mean technique. Statistical mean technique [10] is based on the computed statistics of each and every frame of video or computed statistics of every $\mathrm{k}$ frames by using down sampling to reduce the processing time. In statistical mean technique base, background image is calculated by computing mean of each and every pixel available in video. For $n$ frames and $(x, y)$ is the pixel position of $\mathrm{I}^{\mathrm{th}}$ frame, the summation of all frames computed as shown equation 4 
International Journal of Advanced Information Technology (IJAIT) Vol. 2, No.1, February 2012

$$
X(x, y)=\sum_{i=1}^{n} I(x, y)
$$

The summation of each pixel is in $\mathrm{X}(\mathrm{x}, \mathrm{y})$ of $\mathrm{n}$ frames normalized mean model frame $\mathrm{N}(\mathrm{x}, \mathrm{y})$, is calculated as by dividing each pixel position with $n$, number of frames as shown in equation 5

$$
N(x, y)=X(x, y) / n
$$

Compare this mean model $\mathrm{N}(\mathrm{x}, \mathrm{y})$ to each and every frame from 1 to $n$. For every pixel (x, y) of frame I, compare that pixel position with pixel position $(\mathrm{x}, \mathrm{y})$ of normalized mean model frame $N$ as shown in equation 6

$$
|N(x, y)-I(x, y)|>\tau
$$

where $\tau$ is a predefined threshold value. If the current pixel $(\mathrm{x}, \mathrm{y})$ of frame $\mathrm{I}$, fall in this category than specify it as foreground object pixel otherwise specify as background pixel.

Although statistical mean technique gives good result, but something it suffers by the problem of superfluous effects of foreground objects. Whenever, object or more than one objects remains in video for long period of time, for example, an object is presents in half of the number of frames out of total $n$ number of frames. This leads to erroneous mean model, so that the precise result of moving object detection will not be achieved. This erroneous result is created because of the superfluous effect of that object. To overcome the problem of superfluous effect of the object, modified statistical mean technique is suggested as shown in the following section.

\section{Modified Statistical Mean Method with Noise Removal}

In this section, we present modification to statistical mean method discussed in the previous section. The steps for the modified statistical mean method with noise removal are as follows:

1. Find out incremental statistical mean model initially

2. For each $\mathrm{k}^{\text {th }}$ (initially $\mathrm{k}=1$ ) video frame

3. Compare incremental statistical mean model with $\mathrm{k}^{\text {th }}$ frame

4. Differentiate Foreground and Background Pixels

5. Low pass filters are used as "Pixel Level Process" for reducing blurring effect as pixel level noise reduction.

6. The morphological operations are applied as "Detection of Connected Regions" to find out connected region of the objects.

7. In "Region Level Process", the erroneous object segmentation is removed by applying threshold on to the properties of the region.

8. Extract all the available regions as Moving objects

9. Update incremental statistical mean model and repeat from step 2

\subsection{Modified Statistical Mean Method}

In modified statistical mean technique which we also call incremental statistical mean technique, initially take $k$ number of frames out of total $n$ frames to calculate mean model $N$. This mean model $N$ is used for detection of moving object for first frame. As the first frame is processing, simultaneously update mean model $N$, by taking next frame in derivation of mean model as shown in equation 7 . Here, next frame is $\mathrm{I}_{(\mathrm{k}+1)}$ to update the mean model.

$$
N_{\text {new }}(x, y)=\left(N_{\text {old }}(x, y) * k+I_{k+1}(x, y)\right) / k+1
$$


This updated mean model $\mathrm{N}_{\text {new }}$ is used to for further processing to find the moving object detection. This process is repeated until it reaches to $\mathrm{n}$, total number of frames.

\subsection{Pixel level Processing}

The methods discussed above are generally not sufficient to get desired moving object detection output. Generally, it affects by various noise factors. To overcome this dilemma of noise, it requires further pixel level processing.

We use low-pass filter for the pixel map that leads to eliminate noise[11].Our objective in the implementation of these operations is the removal of noise pixels in the foreground that does not correspond to regions leading real and remove noisy pixels near the bottom and in the regions covered by the pixels which is first plane background.

A Gaussian filter smoothing an image by calculating a weighted average filter coefficient[12]. Gaussian filter modifies the input signal by convolution with a Gaussian function. In two dimensions, Gaussian filter is the product of two such Gaussians, one per direction as described in equation 8

$$
g(x, y)=\frac{1}{2 \prod \sigma^{2}} e^{-\left(\frac{x^{2}+y}{2 \sigma^{2}}\right)}
$$

where, $\mathrm{x}$ is the distance from the origin in the horizontal axis, $\mathrm{y}$ is the distance from the origin in the vertical axis standard deviation of the Gaussian distribution. Fig. 2 shows the frequency response of the 2D Gaussian filter

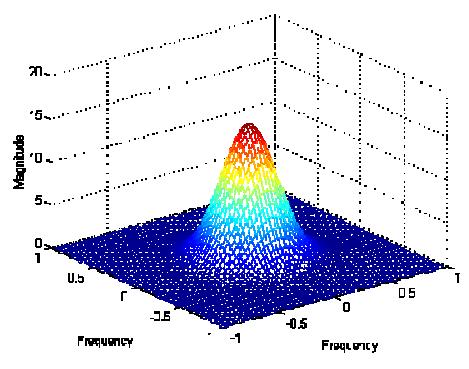

Figure 2: Frequency response of the 2D Gaussian filter

Our aim in applying these operations is removing noisy foreground pixels that do not correspond to actual foreground regions, and to remove the noisy background pixels near and inside object regions that are actually foreground pixels.

\subsection{Finding Connected Regions}

In this phase the filtered foreground pixels are grouped into connected regions. The subsequent erosion dilation morphological operations are applied to find out connected region of the objects. 


\subsection{Region Level Process}

As the elimination of noise at the pixel level, a few small areas are artificial because of bad segmentation. This erroneous object segmentation are removed by applying threshold on to the properties of the region.

\section{Experimental Results}

We tested our method incremental statistical mean method on various sequences of videos acquired on outdoor environments. Figure 3 shows the output of the temporal difference method, by applying the operation as described above, under the outdoor environment. Figure 3 (a) shows the frame sequence 106 to 109 and figure 3 (b) shows frame sequence 107 to 109 of the detected object after applying temporal difference technique as described, in section 2. For example, shown in figure 1 , the value of threshold $\tau$ is 30 .
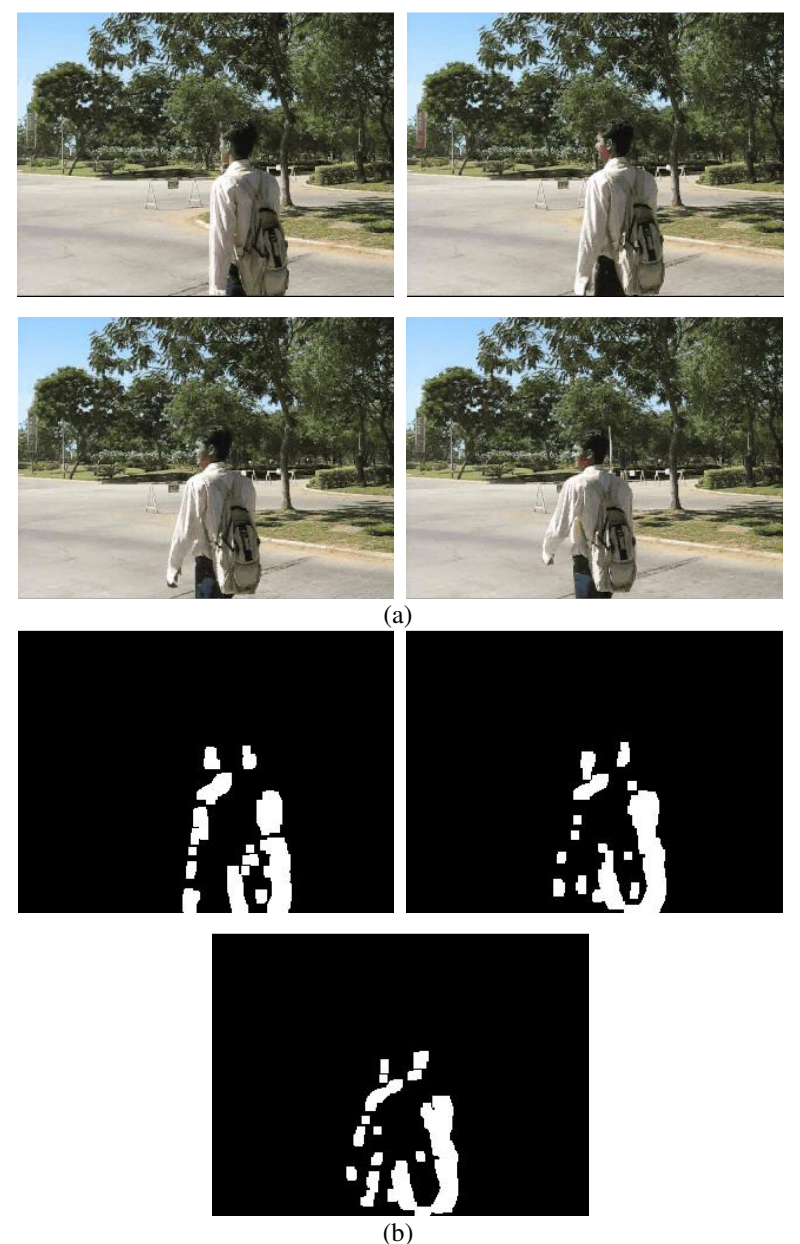

Figure 3: Temporal Difference for moving object detection, (a) input video frame sequence 106 to 109, (b) Temporal Difference result sequence 107 to 109

As described in section 2, it may leave holes in foreground object detection as shown in result. As stated it requires the support of another algorithm to get accurate output. 
Figure 4 shows, the output by applying the incremental statistical method to the video sequence acquired outdoor environment. It provide, detection of moving object accurate, it is able to detect moving object under the dynamic change in the background stationary model. For the example shown in figure 4 , the value of threshold $\tau$ is 30 .
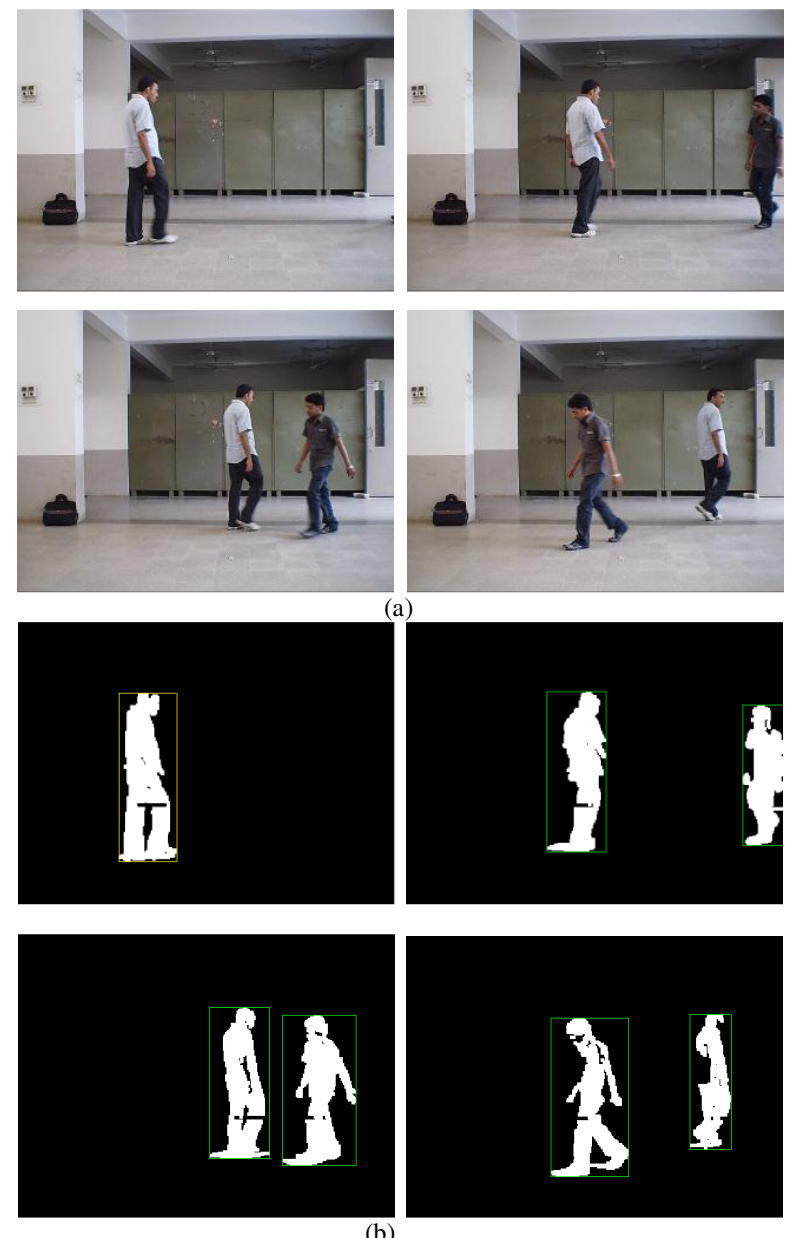

Figure 4: Incremental statistical mean Technique, (a) Input video frame sequence 101111121131 (c) Moving object detected sequence 101111121131

Figure 4 (a) input frame sequence 101111121131 of the video, and 4 (b) shows the output frame sequence 101111121131 that contains moving object detected using the incremental statistical mean method, by using the equation specified in the above section.

Figure 5 shows the results after removing the noise from the video frames. Figure (a) shows input video frames of video 1 and video 2, (b) shows moving object without applying the noise removal methodology and (c) shows the effective result moving object after removing the noise.

We have applied this technique on 12 video, indoor as well as the outdoor environment, and gives accurate result for those. Though, incremental statistical mean technique, achieves good result for moving object detection, it have some disadvantages as, it cannot deal with sudden rain or snow fall, in the video. It cannot differentiate the rain drops and moving object from the video frames. This technique 

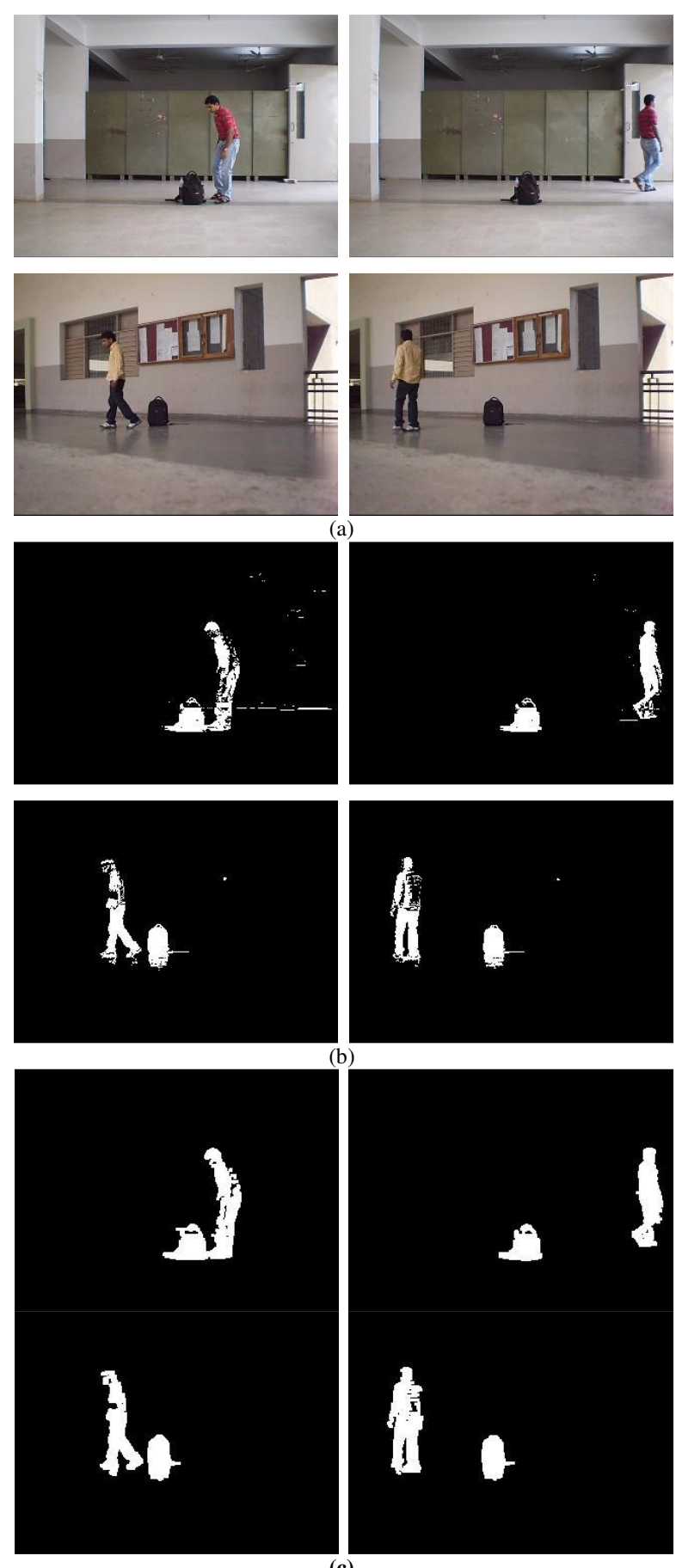

Figure 5: Noise Removal Results (a) Input video frames of video 1 and video 2 (b) Output of object detection without removing noise (c) Output of object detection after removing noise

requires parallel processing to reduce the time complexity. If the value of threshold is not chosen appropriate, to the environment, it may produce the inaccurate result. 
International Journal of Advanced Information Technology (IJAIT) Vol. 2, No.1, February 2012

\section{CONCLUSION}

Moving object detection is the first and low-level step of any visual surveillance system. The traditional moving object detection techniques, with its pros and cons have been explained in brief. These drawbacks are also described experimental results. We have presented a modified statistical mean technique with noise removing process that is computationally fast, by allowing the parallel processing. Modified statistical mean technique, overcome the drawbacks of traditional approach of moving object detection. The noise remove process removes the noise caused due to camera, reflectance noise and gives effective moving objects, as well as provides effective results in the change of light during the day and night, the model of background is changed.

\section{References}

1. Elhabian S. Y., El-Sayed K. M.: Moving object detection in spatial domain using background removal techniques- state of the art, Recent patents on computer science, Vol 1, pp 32-54, Apr, 2008.

2. McIvor A. M.: Background subtraction techniques. Proc. of Image and Vision Computing, 2000.

3. In Su Kim, Hong Seok Choi, Kwang Moo Yi, Jin Young Choi, and Seong G. Kong. : Intelligent Visual Surveillance - A Survey. International Journal of Control, Automation, and Systems (2010) 8(5):926-939

4. Heikkila J. and Silven O.: A real-time system for monitoring of cyclists and pedestrians. Proc. of Second IEEE Workshop on Visual Surveillance, pages 74-81, JUNE 1999.

5. Lipton A. J., Fujiyoshi H., and Patil R. S, : Moving target classification and tracking from real-time video, Proc. of the IEEE Workshop Applications of Computer Vision, pp. 8-14, 1998.

6. Mathew R., Yu Z., Zhang J. : Detecting new stable objects in surveillance video, Proc. of MSP 2005, pp. 1-4

7. Liao H.H., Chang J.Y., Chen L.G. : A localized Approach to abandoned luggage detection with Foreground -Mask sampling, Proc. of AVSS 2008, pp. 132-139.

8. Bayona Alvaro, SanMiguel Juan C., Martínez Jose M. : Stationary Foreground Detection using Background Subtraction and Temporal Difference in Video Surveillance. Proceedings of 2010 IEEE 17th International Conference on Image Processing September 26-29, 2010.

9. Yilmaz, A., Javed, O., and Shah, M. 2006.: Object Tracking: A survey. ACM Comput. Surv. 38, 4, Article 13, Dec 2006.

10. Kotikalapudi U. K. Abnormal event detection in video, M.Tech Thesis, Indian Institute of Science, Banglore, July 2007.

11. Gonzalez R. C. and Woods R. E., Digital Image Processing (third edition), Reading, Massachusetts:Addison-Wesley, 1992

12. Sapiro G. and Ringach D. L. , "Anisotropic diffusion of color images", In Proc. SPIE, 1996,2657:471-382. 